$4-2001$

\title{
Modeling Nonlinear Behavior in a Piezoelectric Actuator
}

\author{
Hanz Richter \\ Cleveland State University, h.richter@csuohio.edu \\ Eduardo A. Misawa \\ Oklahoma State University, misawa@okstate.edu
}

D. A. Lucca

Oklahoma State University

H. Lu

Oklahoma State University

Follow this and additional works at: https://engagedscholarship.csuohio.edu/enme_facpub

Part of the Mechanical Engineering Commons

How does access to this work benefit you? Let us know!

\section{Publisher's Statement}

NOTICE: this is the author's version of a work that was accepted for publication in Precision Engineering. Changes resulting from the publishing process, such as peer review, editing, corrections, structural formatting, and other quality control mechanisms may not be reflected in this document. Changes may have been made to this work since it was submitted for publication. A definitive version was subsequently published in Precision Engineering, 25, 2, April 2001, DOI: 10.1016/S0141-6359(00)00067-2

\section{Original Citation}

Richter, H., Misawa, E. A., Lucca, D. A., 2001, "Modeling Nonlinear Behavior in a Piezoelectric Actuator," Precision Engineering, 25(2) pp. 128-137.

This Article is brought to you for free and open access by the Mechanical Engineering Department at EngagedScholarship@CSU. It has been accepted for inclusion in Mechanical Engineering Faculty Publications by an authorized administrator of EngagedScholarship@CSU. For more information, please contact library.es@csuohio.edu. 


\title{
Modeling nonlinear behavior in a piezoelectric actuator
}

\author{
H. Richter, E.A. Misawa*, D.A. Lucca, H. Lu ${ }^{1}$ \\ School of Mechanical and Aerospace Engineering, Oklahoma State University, Stillwater, OK 74078-5016, USA
}

\section{Introduction}

Piezoelectric actuators have become a standard option in positioning applications where the displacements must be small and highly accurate. In particular, ultra-precision manufacturing requires exceptionally fine and repeatable motions, making piezoelectric actuators a common choice. These actuators, however, are sensitive to environmental changes such as temperature; and moreover, several nonlinearities are present in the behavior. The well-known phenomena of hysteresis and creep, along with nonlinear voltage dependence affect the dynamic response, sometimes precluding closed-loop operation [1]. Consequently, a precise mathematical model is necessary to design feedback controllers properly. This work is motivated by a particular problem related to ultra-precision manufacturing. A nanometric cutting instrument, denoted as Nanocut, was designed and built as a team effort by Oklahoma State University and the University of North Carolina at Charlotte, as a means to investigate the mechanics of ultra-precision machining [2]. The instrument employs a tubular piezoelectric actuator which achieves sub-nanometer resolution for $3 \mathrm{D}$ positioning, but not necessarily highly accurate motions 7873.

* Corresponding author. Tel.: +1-405-744-5904; fax: +1-405-744-

E-mail address: misawa@okstate.edu (E.A. Misawa). when operated in open loop. This is primarily due to the associated nonlinear effects, which can cause errors on the order of microns, while environmental perturbations, such as temperature changes, cause smaller errors when experiments are carried out in a controlled environment. The present work is focused on the axial extension of the piezoelectric actuator, hereafter referred to as "PZT tube". A non-formal analogy between the observed behavior and that of nonlinear viscoelastic materials is established. The motivation for such an analogy is a remarkable similarity observed in the input/output behavior of the piezo tube and nonlinear viscoelastic materials under uniaxial extension. This analogy is used as a basis to construct the proposed differential model. An extensive set of experiments is carried out to estimate the parameters of the model. The experiments involve both frequency and time domain measurements, which are used to cross-examine the model for self-consistency between domains. Finally, an independent set of experiments is performed to validate the numerical model.

Piezoelectric materials and actuators have received considerable attention in the literature, especially in recent years, with the advent of new applications based on scanning-probe microscopy. Instruments such as the scanning tunneling and atomic force microscope almost universally use piezoelectric tube actuators to generate the fine motions required [3]. In recent years PZT actuators have also been 
introduced to high-density hard disk applications as a subactuator for fin head displacement [4]. Much of the published research, however, is concerned only with models based on the linearized constitutive laws of piezoelectric behavior. The most widely accepted linear description of piezoelectric materials is given in the IEEE Standard on Piezoelectricity published in 1987 as an American National Standard [5]. In many instances, actuator geometry and simple loading conditions -the term "loading" understood as imposed stress and electric field result in simple second order linear transfer functions that map applied voltage to actuator displacement ([8], [7], [9], [10] among many others.) Considerable research works have been reported in the literature that describe the linear portion of the behavior of piezoelectric actuators, especially for the stacked-type construction. The fundamentals of nonlinear piezoelectric phenomena are analyzed mostly in the framework of the physics of crystals and thermodynamics, rendering descriptions that are extremely difficul to reduce to a set of nonlinear ordinary differential equations [11]. As stated in [12], basing model derivation on the IEEE linear constitutive relations requires several assumptions, resulting in an oversimplifie description that fails to capture the essential nonlinear behavior that is present in all piezoelectric ceramics. In the case of PZT tube actuators, perhaps the highest degree of simplificatio is given in [13], where the axial motion is reduced to the description

$$
u_{z z}=d_{33}^{\text {eff }} \frac{V}{L}
$$

where $u_{z z}$ is the axial strain, $d_{33}^{\text {eff }}$ is an effective piezoelectric constant that considers tube geometry and an average electric fiel value, $V$ is the applied radial voltage, and $L$ is tube length. From an engineering modeling perspective, several works have been reported that specificall address the nonlinearities, for example, Goldfarb and Celanovic [12] utilized a Maxwell slip model to describe hysteresis. Another work [21] that focuses on the modeling of hysteresis adapts the Preisach mathematical model to the case of piezoelectric actuators, with good agreement between model and experiment. In several works ([7], [19]) hysteresis is accounted for by augmenting the linear dynamics with an empirical differential equation of hysteretic behavior. The main disadvantage of such an approach is the cumbersome mathematical expressions which make it difficul to fin a set of parameters that can match experimental data. An interesting approach was taken by Tamer [3]. In that work, the linear dynamic description is augmented in the frequency domain by a describing function of a hysteresis nonlinearity, rendering a model that is still linear, but includes the phase-lag effects due to the actual nonlinear phenomenon. Thus, better controllers can be designed, even though the model may not be accurate in the time domain. This is presented as an $a d-h o c$ solution to a particular problem rather than as a general treatment. The creep phenomenon that is observed

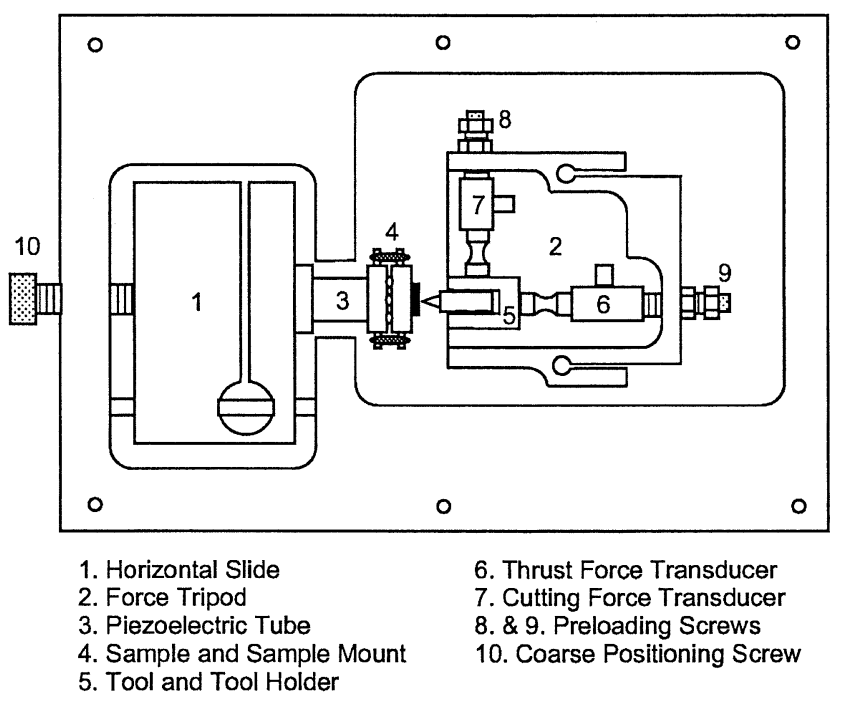

Fig. 1. The Nanocut instrument.

in piezoelectric materials has been treated in fewer publications. Moreover, these works mostly deal with descriptions of creep that are not intended to serve as models for control purposes [14]. Similarly, the nonlinear dependence of axial displacement on input voltage has not received much attention. Arguably, a reason for these voids is the fact that both effects may be easily compensated by a feedback controller, since the creep can be regarded as a slow output disturbance and the nonlinear dependence on input voltage as a gain uncertainty. A simple approach to the compensation of creep and hysteresis by inserting a capacitor in series with the piezoelectric actuator was proposed by Kaizuka and Siu [6]. In fact, controllers are now commercially available that achieve subnanometer resolution and accuracy for unidirectional actuators. A nonlinear threedimensional model that encompasses creep, nonlinear voltage dependence, and hysteresis would constitute an important contribution to the development of high precision piezoelectric tube actuators.

\section{Observed behavior}

In this section, the experimental setup used throughout the research is described first Then, three nonlinear effects are identifie from experimental results.

\subsection{Experimental setup}

The piezo actuator is used as a sample-positioning device in the Nanocut instrument. The instrument is capable of three-dimensional positioning of a sample with respect to a stationary cutting tool or indenter, and is equipped with force and displacement transducers which transmit data into a computer for later analysis. Fig. 1 illustrates the mechanical hardware as viewed from the top. The piezoelectric tube 


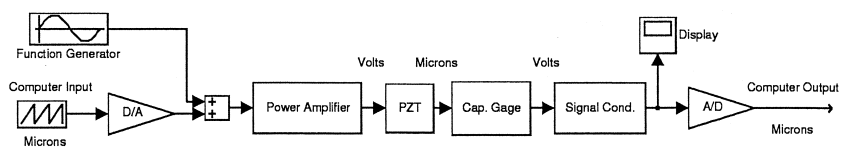

Fig. 2. Functional Diagram.

(3) is mounted on the horizontal slide (1) as a cantilever beam. The application of voltage to the appropriate electrodes results in either horizontal/vertical tip displacement by bending, or longitudinal expansion of the piezoelectric tube. A capacitance gage is positioned inside the PZT tube to measure changes in longitudinal motion. The piezoelectric material employed in the actuator is PZT-5 (LeadZirconate-Titanate). ${ }^{2}$ Fig. 2 shows a functional diagram of the open-loop $z$ positioning system. The commanded position in microns is sent to a 16 bit D/A board on a PCcompatible computer. The resulting analog voltage is amplifie and applied to the $z$ electrode of the PZT tube and the expansion measured by the capacitance gage is amplifie to be read by the 16 bit A/D board. The capacitance gage and its signal conditioner have a combined sensitivity of $3.96 \mathrm{~V}$ $\mu m^{-1}$, resulting in a A/D resolution of $0.077 \mathrm{~nm} / \mathrm{bit}$. The PZT tube has a nominal ${ }^{3}$ sensitivity of $8.33 \mathrm{nmV}^{-1}$, and a maximum displacement of $1.5 \mu \mathrm{m}$, imposed by the construction of Nanocut. This implies a maximum of $180 \mathrm{~V}$ between electrodes. The tube and power amplifie have an average combined sensitivity of $0.15 \mu \mathrm{m} V^{-1}$, resulting in a $\mathrm{D} / \mathrm{A}$ resolution of $0.046 \mathrm{~nm} / \mathrm{bit}$. These resolutions are far from being fully effective in practice due to environmental perturbations and the nonlinear effects under study. A more detailed description of Nanocut is reported elsewhere [2].

\subsection{Three nonlinear effects}

\subsubsection{Nonlinear gain}

When several constant voltage inputs are applied to the $z$-electrodes, the corresponding responses are not proportional to the applied voltage. A set of experiments was performed to evidence the effect. Power amplifie transient effects were eliminated from the experiment by using a switch, to better simulate a step input. For each input voltage, ranging from 15 to 120 Volts, data were recorded at two different resolutions. Fig. 3 (left) represents short-term behavior, sampled every $5 \mu$ s, i.e., at $200 \mathrm{kHz}$. The plot on the right shows the same experiment, with data sampled every $500 \mu \mathrm{s},(2 \mathrm{kHz})$, to evidence the slow creep phenomenon. In both cases the response has been normalized by dividing by the input value. Physically, the source of nonlinearity in this case is the voltage-dependency of the piezoelectric constants. A damped oscillation near $12 \mathrm{kHz}$ is observed in the response. This is possibly a structural mode of the PZT tube, not to be modeled in this work.

\subsubsection{Nonlinear creep}

The steady creep feature is evident in Fig. 3 (right). The rate of creep is sensibly constant for each input voltage, up
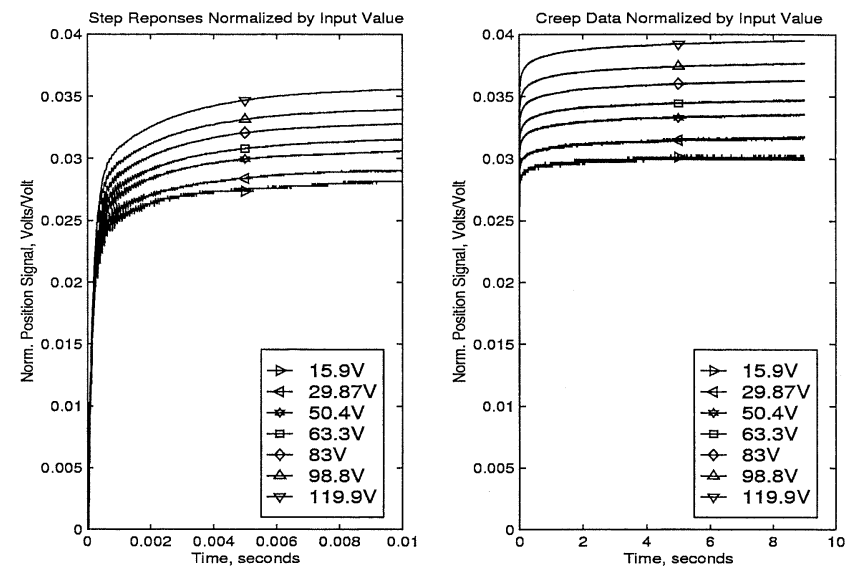

Fig. 3. Nonlinear Response to a series of step voltage inputs.

to the time-frame of the experiment (about 10 seconds). Physically, creep is caused by a gradual alignment of dipoles, and therefore expansion -and not rate of expansionshould converge to a steady value. Convergence is achieved, however, over a period of time that is much larger than the time constant of any practical control system. Creep is deemed to be nonlinear based on the fact that expansion rates depend on voltage in a nonlinear fashion, as will be shown later.

\subsubsection{Frequency behavior and hysteresis}

A set of responses corresponding to sinusoidal inputs of several amplitudes and frequencies was recorded. The input frequency is observed to dominate the response up to the firs few kilohertz. Significan distortion and appearance of harmonics is observed beyond $5 \mathrm{kHz}$. Fig. 4 shows the magnitude and phase of the fundamental frequency component. The data represented correspond to a experimental sinusoidal input describing function, or SIDF. A nonlinear gain characteristic is clear in this representation. Fig. 5
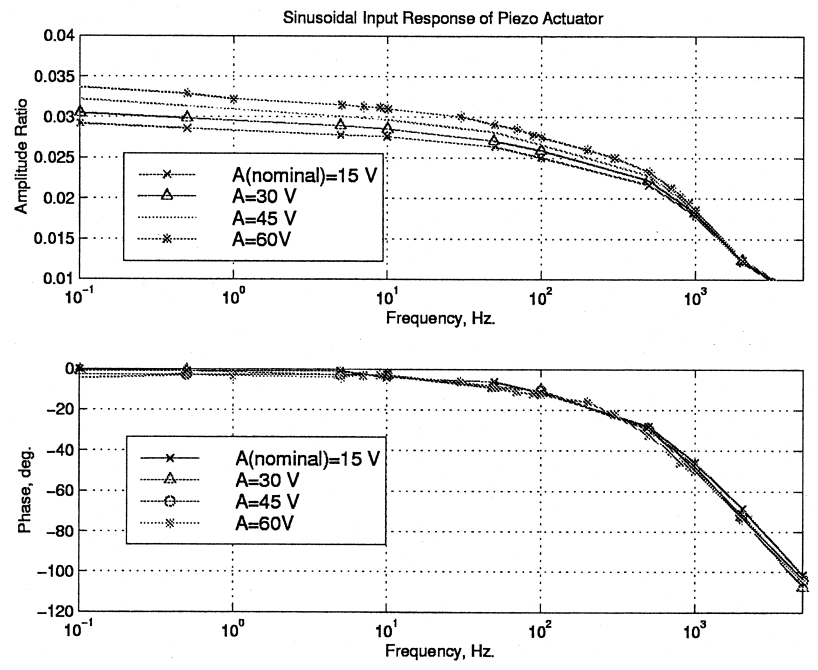

Fig. 4. Sinusoidal Input Response. 


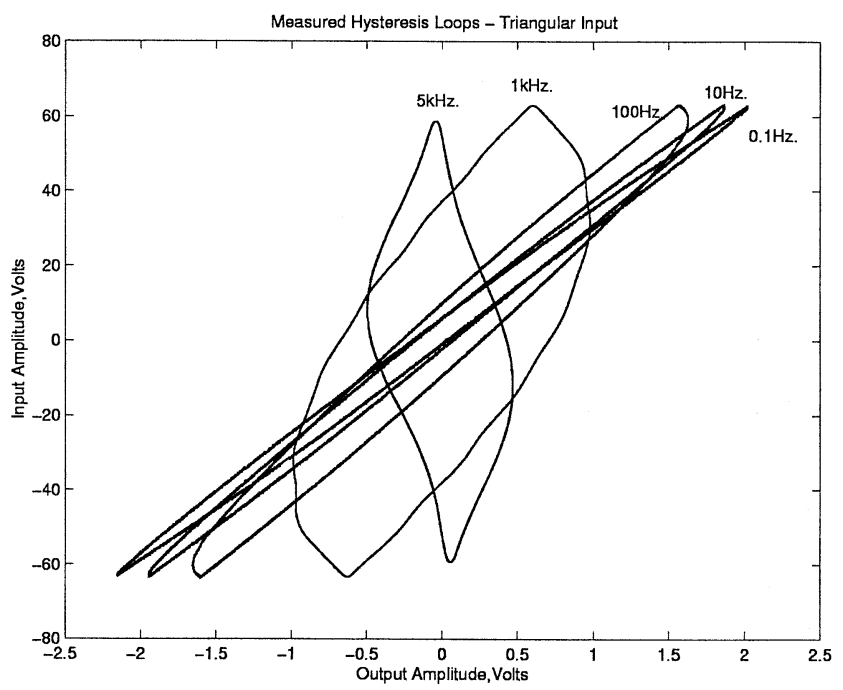

Fig. 5. Experimental Hysteresis Loops.

shows a set of experimental hysteresis loops. A $60 \mathrm{~V}$ triangular voltage at several frequencies was applied to the actuator electrodes. As discussed next, phase lag due to linear dynamics is embedded in the loops, but the presence of rate-independent hysteresis makes the effect nonlinear as a whole.

\subsubsection{Hysteresis in context: some important statements}

There is no complete agreement in the use of the term "hysteresis" among the various research field where memory effects are present. In the case of piezoelectric actuators, only a few works explicitly point out the nature of the memory effect that is under consideration [12]. The term "hysteresis" is usually attributed to rate-independent memory, although some material has been published in which the term is employed to describe rate-dependent phenomena [15]. Some other publications employ the terms static hysteresis and dynamic hysteresis [20] to distinguish the two effects. An important observation is the fact that rate-independent effects are not found isolated from rate-dependent memory, except in idealized situations. The relative importance of the two effects should be assessed before including either in a mathematical model. In piezoelectric actuators and in many other engineering systems, it is found that the rate-dependent memory has a greater importance at high frequencies, because of the common phase-lag characteristics of most physical devices. Rate-independent phenomena prevails at low frequencies for the same reasons. In fact, it is a common practice to reveal the effect in experiments by applying slow cyclic inputs, so that the phase lag due to rate-dependent memory is negligible. This practice may lead to erroneous results, however, when the system is only linear, but contains poles at or near the origin. In this case, for sufficientl slow inputs, a loop is obtained which may be erroneously regarded as hysteretic. For the case of the present PZT tube, the phase-lag due to the linear portion of

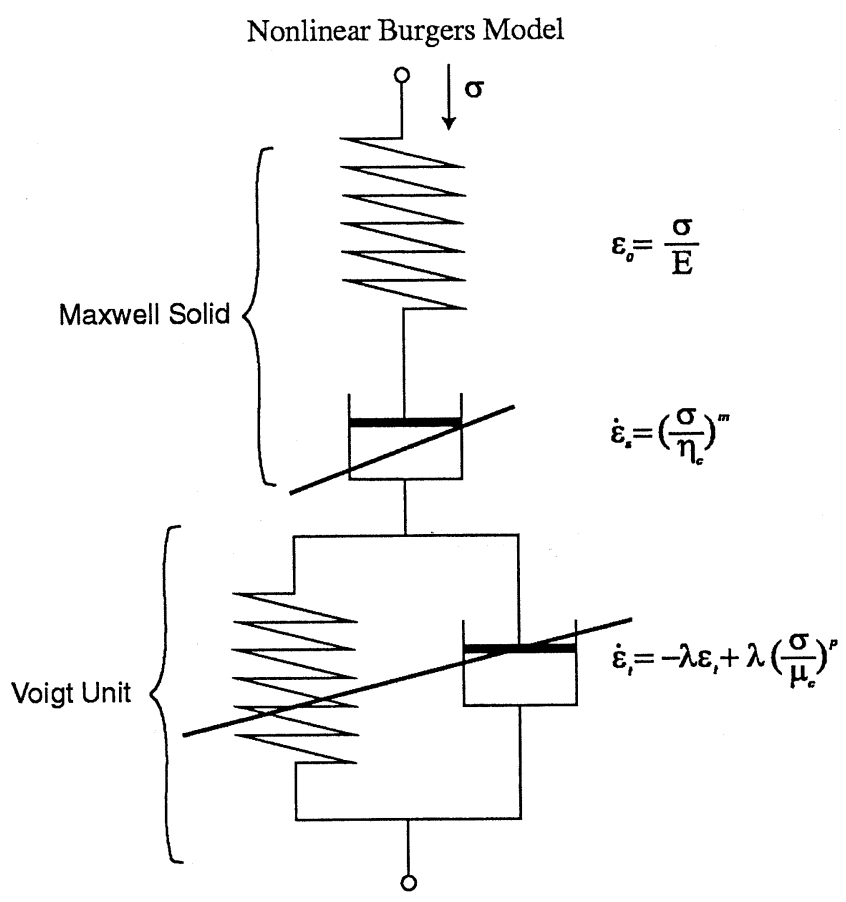

Fig. 6. Simple Nonlinear Burgers Model.

the dynamics is seen to prevail over most of the frequency range of interest. It is because of this observation that the term "hysteresis loop" is used throughout this work to refer to the coupled memory effect. The proposed model, being of a viscoelastic type, does not account for rate-independent memory. It does provide sufficientl accurate predictions of the coupled hysteresis loops, however.

\subsection{Models for nonlinear viscoelastic behavior}

A large number of models have been proposed and tested over the years that explain the observed phenomena to a certain degree of accuracy, but there is no all-embracing theory which is likely to accommodate all eventualities [17]. As in other fields models can be constructed by empirical observation or by physical considerations. Physical models for nonlinear viscoelastic behavior are still in development, and their reduction to a set of manageable ordinary differential equations constitutes a difficul task. For this reason, a phenomenological model for nonlinear viscoelasticity is employed as a starting point. The model presented here can be traced back to the work of Marin and Pao, [16]. Even though the theory is based on empirical observation of the stages of creep response when a constant stress input is applied, the resulting model can still be linked to a nonlinear Burgers rheological model $[16,18]$. The Burgers model in the linear case is of wide application in linear viscoelasticity, and its parameters are physically welldefine quantities, such as viscosity and elastic modulus. The equations for variable input represent the behavior of a nonlinear spring-dashpot system, as shown in Fig. 6. In the 
linear case, this is known as the four-element Burgers model. In Fig. 6 the linear spring corresponds to an instantaneous elastic response. The single nonlinear dashpot relates to the steady creep component for constant stress, and the parallel arrangement of a nonlinear spring and dashpot represents a transient component. ${ }^{4}$ This parallel arrangement, in the linear case, is known as a Voigt element.

\subsection{A non-formal analogy}

The input/output nonlinear features observed in the piezoelectric actuator match -at least in a qualitative sensethose observed in nonlinear viscoelastic materials under uniaxial extension. In order to construct a model, stress and strain in the viscoelastic case can be interpreted as voltage and longitudinal expansion, respectively. A differential model that describes the basic Burgers model represented in Fig. 6 is given as

$$
\begin{aligned}
& \dot{\varepsilon}_{s}=\left(\frac{\sigma}{\eta_{c}}\right)^{m} \\
& \dot{\varepsilon}_{t}=-\lambda \varepsilon_{t}+\lambda\left(\frac{\sigma}{\mu_{c}}\right)^{p} \\
& \varepsilon=\varepsilon_{s}+\varepsilon_{t}
\end{aligned}
$$

where $\varepsilon_{s}$ and $\varepsilon_{t}$ represent the steady and transient components of the total creep, $\varepsilon$ that is the response to an applied stress $\sigma$. Note that when $p=1$, the spring and dashpot in the linear Voigt element represent an elastic modulus $\mu_{c}$, and a viscosity coefficien $b=\mu_{c} / \lambda$. Similarly, in the linear case, when $\mathrm{m}=1, \eta_{c}$ represents a viscosity coefficient When $p>1$, as usually found experimentally, the parallel arrangement must be treated as a "black-box", and it is not possible nor relevant to fin isolated parameters that correspond to viscosity and elasticity, in a strict sense. However, if $p$ is close to one, as it is usually the case, the physical significanc of $\mu$ and $\mu / \lambda$ is maintained for practical purposes. The same arguments apply to the nonlinear dashpot representing steady creep.

Upon application of the analogy, nonlinear dynamic behavior of the PZT tube can be treated as nonlinear viscoelastic response, which has received more attention than the piezoelectric case. Of course, the analogy is of limited use in gaining physical insight about the piezo actuator, but once more, the goal is to produce a good control model. This approach is conceptually similar to the one proposed by Goldfarb and Celanovic [12], replacing the elasto-slide elements by Voigt units. The present approach has the advantages of being able to capture the observed creep effect, which is ignored in [12], as well as the nonlinear dependence on input voltage.

\subsection{The proposed model}

The model consists of a series arrangement of $n$ Voigt units and a nonlinear dashpot representing nonlinear creep. The equations presented here result from extending and modifying the equations for nonlinear viscoelasticity presented in [18]. The derivations are rather simple, but too lengthy to be included in this paper. Using the $n$ transient components and the steady creep component as states, the model equations can be written in state-space form. Note that the states are completely decoupled.

$$
\left\{\begin{array}{l}
\dot{\varepsilon}_{v_{1}}=-\lambda_{1} \varepsilon_{v_{1}}+\lambda_{1} r_{1} f_{t}(V(t)) \\
\dot{\varepsilon}_{v_{2}}=-\lambda_{2} \varepsilon_{v_{2}}+\lambda_{2} r_{2} f_{t}(V(t)) \\
\vdots \\
\dot{\varepsilon}_{v_{n}}=-\lambda_{n} \varepsilon_{v_{n}}+\lambda_{n} r_{n} f_{t}(V(t)) \\
\dot{\varepsilon}_{s}=f_{s}(V(t))
\end{array}\right.
$$

with output equation

$$
\varepsilon=\varepsilon_{v 1}+\varepsilon_{v 2}+\cdots+\varepsilon_{v_{n}}+\varepsilon_{s}
$$

Also, the following restriction is imposed

$$
\sum_{i=1}^{n} r_{i}=1
$$

In the above equations, $\lambda_{i}$ and $r_{i}$ are characteristic frequencies and weights, respectively. The "transient" response to a step input then becomes a weighted sum of exponentials which converges to unity, with a common nonlinear gain represented by the function $f_{t}(V(t))$. The overall response is composed of the transient response and a permanent creep represented by the last state. Power functions of voltage are to be tested for experimental validity. Note that when the exponents are non-integer values, which are likely to arise from the parameter estimation procedures, the power functions are complex-valued. To avoid this inconvenience, the following modificatio is introduced:

$$
\begin{aligned}
& f_{t}(V)=\operatorname{sign}(V)\left(\frac{|V|}{\mu}\right)^{p} \\
& f_{s}(V)=\operatorname{sign}(V)\left(\frac{|V|}{\eta}\right)^{m}
\end{aligned}
$$

where $\operatorname{sign}(V)=|V| / V$ denotes the signum function. This, however, implies that the step response must be an odd function of $V$, because $f(-V)=-f(V)$ for the chosen power functions. This was experimentally confirme by applying negative and positive steps of the same magnitude and comparing the outputs.

\subsubsection{Computation of the sinusoidal input describing function (SIDF)}

The SIDF of the model is found to be particularly useful for cross-verificatio between time and frequency domains, as described later. The SIDF is provided below 


$$
\begin{aligned}
& N(A, w)=N_{s}(A, w)+\sum_{i=1}^{n} N_{i}(A, w), \text { where: } \\
& N_{i}(A, w)=\frac{A^{p-1}}{\mu^{p}} \frac{2 \Gamma(p / 2+1)}{\sqrt{\pi} \Gamma(p / 2+3 / 2)} \frac{\lambda_{i} r_{i}}{\lambda_{i}+j w} \\
& N_{s}(A, w)=\frac{A^{m-1}}{\eta^{m}} \frac{2 \Gamma(m / 2+1)}{\sqrt{\pi} \Gamma(m / 2+3 / 2)} \frac{1}{j w}
\end{aligned}
$$

The sinusoidal input amplitude is denoted by $A$ and $w$ is the frequency. Also, $\Gamma(x)$ is the Gamma (generalized factorial) function.

\subsection{Parameter estimation from time domain data}

Here we assume that model parameters are to be identifie from a series of step-voltage responses. This is the easiest way to estimate parameters using an oscilloscope with data storage capability. In this case, it is assumed that the input functions in the model given by Eq. (6) are power laws of the form

$$
\begin{aligned}
& f_{t}(V)=\left(\frac{V}{\mu}\right)^{p} \\
& f_{s}(V)=\left(\frac{V}{\eta}\right)^{m}
\end{aligned}
$$

It is also considered that there is no "instantaneous" elastic component, as such an approximation is granted only for true viscoelastic materials, in which the subsequent expansion is slower than the initial fast transient by orders of magnitude. The set of parameters to be estimated consists of model order $n+1$, the $n$ characteristic frequencies $\lambda_{i}$ and their weights $r_{i}$, and the parameters from the above power laws, namely, $p, m, \mu, \eta$. These parameters are to be recovered from the step responses and creep data described earlier for seven input voltages. Note that parameter recovery from a step response, which is not a frequency-rich signal, is justifie by the fact that a resonance-free model has been chosen a priori, and the estimation is based on the solutions of the differential equation rather than in the form of the equations themselves. Having completed the parameter estimation, the model given by equations (6) can be readily constructed and tested for inputs other than a step.

Table 1

Measured vs. Predicted Slopes of Steady Creep

\begin{tabular}{lll}
\hline$\eta=2850.37, \mathrm{~m}=1.5313$ \\
\hline Voltage & Measured Slope & Predicted Slope \\
\hline 15.90 & $2.4290 \times 10^{-4}$ & $3.5420 \times 10^{-4}$ \\
29.87 & $1.6688 \times 10^{-3}$ & $0.9302 \times 10^{-3}$ \\
50.40 & $2.0194 \times 10^{-3}$ & $2.0724 \times 10^{-3}$ \\
63.30 & $2.7963 \times 10^{-3}$ & $2.9377 \times 10^{-3}$ \\
83.00 & $4.9799 \times 10^{-3}$ & $4.4485 \times 10^{-3}$ \\
98.80 & $4.7710 \times 10^{-3}$ & $5.8088 \times 10^{-3}$ \\
119.9 & $7.7445 \times 10^{-3}$ & $7.8131 \times 10^{-3}$ \\
\hline
\end{tabular}

Table 2

Measured vs. Predicted Final Values of Transient Component

\begin{tabular}{llll}
\hline$\mu=31.3701, \mathrm{p}=1.1279$ & & \\
\hline Voltage & Final Value & Final Slope & Predicted F.V \\
\hline 15.90 & 0.4766 & Zero, 16 digits & 0.4646 \\
29.87 & 0.9321 & Zero, 16 digits & 0.9462 \\
50.40 & 1.6725 & Zero, 16 digits & 1.7071 \\
63.30 & 2.1714 & Zero, 16 digits & 2.2074 \\
83.00 & 2.9660 & Zero, 16 digits & 2.9964 \\
98.80 & 3.6774 & Zero, 16 digits & 3.6472 \\
119.9 & 4.6681 & Zero, 16 digits & 4.5371 \\
\hline
\end{tabular}

\subsection{Experimental results 1: time-domain estimation}

\subsubsection{Steady creep}

The steady rates of creep are extracted using a regression line fitte to the last portion of the long-term data. The slopes of the regression lines are the constant rates to be fitte to a voltage power law. To fi the obtained slopes to a power law of voltage, another linear regression is applied. The resulting parameters are summarized in Table 1 .

\subsubsection{Transient component}

Once the steady creep is characterized, it is subtracted from the overall creep response, leaving the transient component for parameter identification To verify the constant creep rate assumption, a regression line is fitte to the last portion of the transient components, yielding an estimated slope of zero and the fina steady values summarized in the Table 2, with fina values expressed in Volts. Fig. 7 shows a poor fi for the slopes of steady creep and a very close fi for the fina values. Moreover, it is seen that the slope data presented may not be suitable for approximation by any reasonable, smooth function of voltage. In simulation studies for control system design, this limitation may be circumvented by implementing look-up tables.
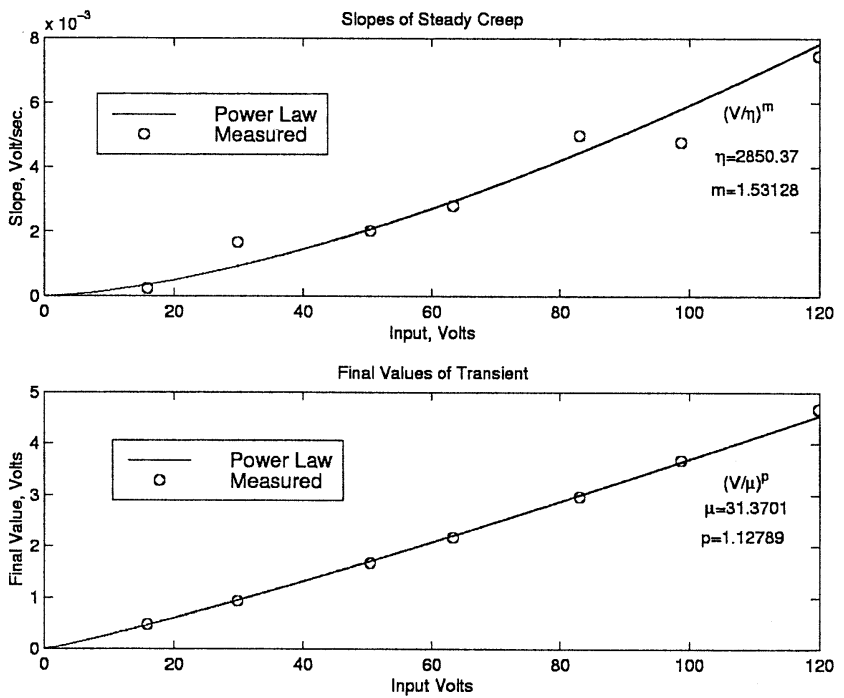

Fig. 7. Fitness of power law parameters. 
Table 3

Estimated Characteristic Frequencies and Weights

\begin{tabular}{llll}
\hline $\begin{array}{l}\text { Char. Freq. } \lambda \\
\text { Proc. X }\end{array}$ & $\begin{array}{l}\text { Weight } r \\
\text { Proc. X }\end{array}$ & $\begin{array}{l}\text { Char. Freq. } \lambda \\
\text { NLSQ }\end{array}$ & $\begin{array}{l}\text { Weight } \\
\text { NLSQ }\end{array}$ \\
\hline 7549.700 & 0.4449 & 7506.799 & 0.6614 \\
1343.200 & 0.0785 & 1365.767 & 0.2322 \\
399.9356 & 0.2790 & 390.7856 & 0.0284 \\
28.26340 & 0.0824 & 73.03461 & 0.0584 \\
4.742100 & 0.0554 & 4.875001 & 0.0322 \\
0.648000 & 0.0591 & 0.632376 & 0.0313 \\
& & $\Sigma \mathrm{r}_{\mathrm{i}}=1.044$ (NLSQ) & \\
\hline
\end{tabular}

\subsubsection{Estimation of characteristic frequencies, weights, and model order}

The last step is to obtain the characteristic frequencies and their corresponding weighting factors from the normalized transient. For this purpose, the transient response obtained from the $83 \mathrm{~V}$ input is chosen, for it has a good signal-to-noise ratio. An iterative process that estimates real characteristic frequencies from a step response known as "Procedure X" [15] is employed to obtain a firs approximation. Six frequencies and weights are found, resulting in a model of order seven. These estimates are employed as initial guesses in a standard nonlinear least-squares routine (NLSQ), providing a set of refine parameters. Notice that the $83 \mathrm{~V}$ normalized data are re-sampled to contain only 1000 logarithmically-spaced points, so that the NSLQ routine is possible to implement. Re-sampling also has a lowpass filterin effect on the data. Table 3 summarizes the obtained parameters. Note that the sum of the weighting factors is close to one ${ }^{5}$, as required, and that some of them are very small, so model reduction may be easy to accomplish by approximation. A comparison between predicted and measured responses to a constant voltage input is shown in Fig. 8. The predicted responses are plots of the analytic solutions to Eq. (6) using the estimated parameters. The
Table 4

Confidenc Intervals for Parameter Estimates

\begin{tabular}{lll}
\hline Parameter & Mean & $95 \%$ Confidenc Interval \\
\hline $\mathrm{p}$ & 1.1279 & {$[1.0948,1.1610]$} \\
$\mathrm{m}$ & 1.5313 & {$[1.0480,2.0145]$} \\
$\nu$ & 2850.37 & {$[1597.12,7255.03]$} \\
$\mu$ & 31.3701 & {$[25.3219,39.3701]$} \\
\hline
\end{tabular}

normalized rms prediction errors range from 0.64 to 2.75 percent. It can be stated that the model, together with its estimated parameters, provides an adequate fi of the observed time-domain data. The $95 \%$ confidenc intervals for the parameters corresponding to the power laws are provided in Table 4. All parameters except $\eta$ have acceptable deviations. The poor fi observed graphically for the creep rate is quantifie by the confidenc intervals.

\subsection{Experimental results 2: time-frequency cross-verification}

It is evident that it is always possible, in principle, to $\mathrm{f}$ nd appropriate voltage and time functions that match the step responses to a good degree of accuracy. Given the model structure, parameter recovery is adequate from a set of step responses, however it is questionable if the structure itself is compatible with the response in the frequency domain. In order to verify this important aspect, the analytical SIDF of the model is computed using parameters estimated from time domain. Then, analytical and experimental SIDF's are compared. The experimental SIDF is obtained by applying sinewaves of various amplitudes and frequencies, and recording the output amplitudes and phase lags for each frequency. Results are shown in Fig. 9, showing a good agreement.
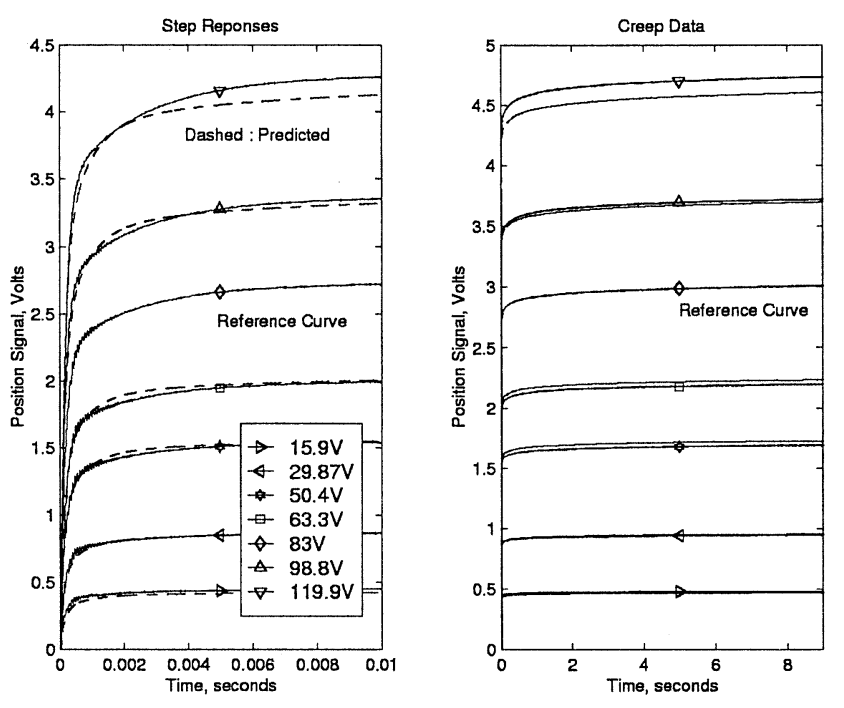

Fig. 8. Prediction of the Step Response.
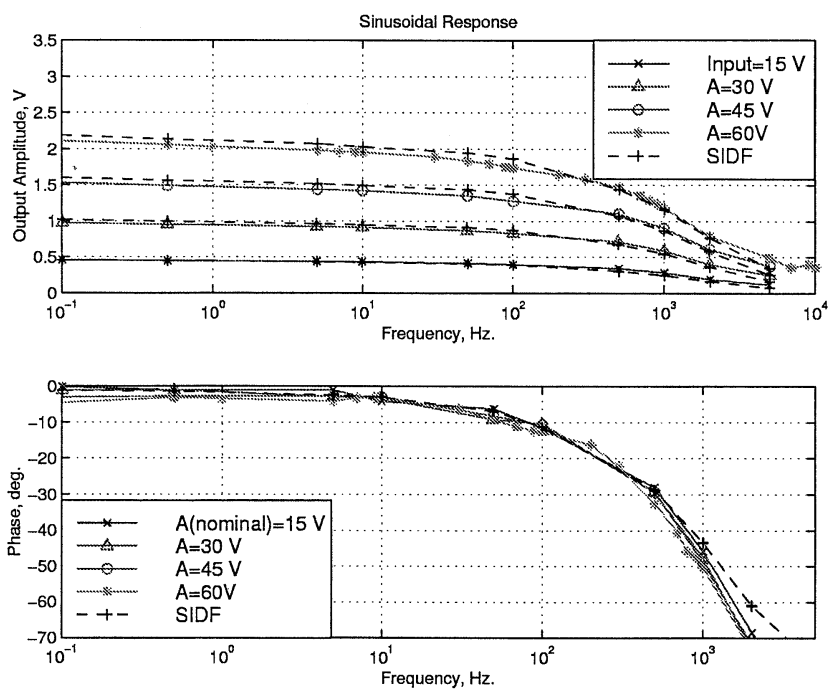

Fig. 9. SIDF Prediction of Sinusoidal Response. 

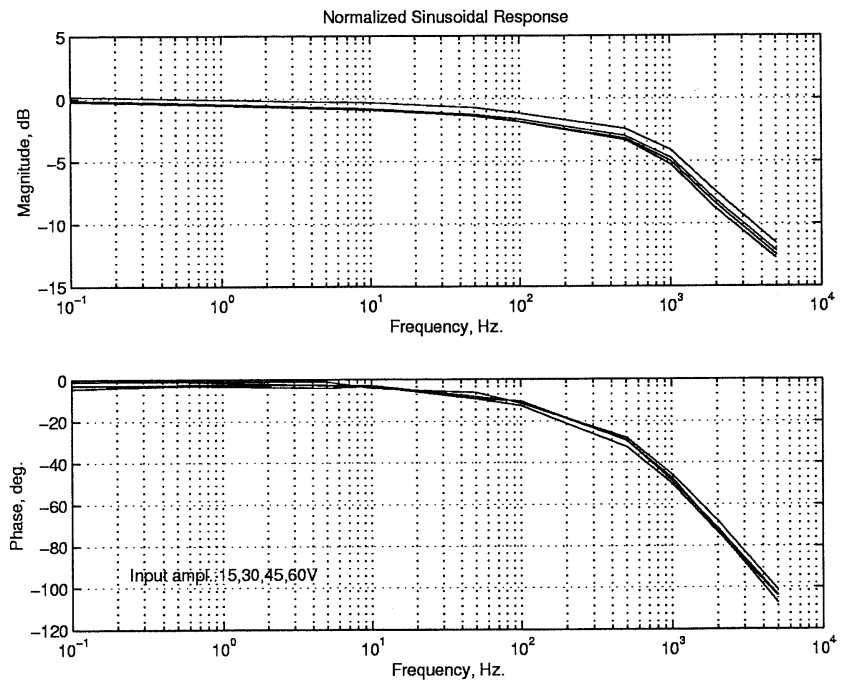

Fig. 10. Normalized Sinusoidal Response.

\subsubsection{An alternative estimation procedure}

The measured magnitude and phase are converted into complex form for each of the amplitudes and frequencies under study. Then, in a manner analogous to removing the steady creep from time-domain data, the creep portion of the SIDF is removed from the complex data. This is possible because parameters $p, m, \mu, \eta$ are available from estimation in time. Then, the remaining data are normalized by the SIDF coefficien that corresponds to the transient component. The result should be a set of data that fully matches in frequency, phase, and magnitude. Also, the magnitude should asymptotically approach unity (or $0 \mathrm{~dB}$ ) as frequency approaches zero. This is demonstrated in Fig. 10. Note that such complex data constitutes the Bode plot of a linear transfer function in partial fraction expansion form:

$$
G(s)=\sum_{i=1}^{n} \frac{\lambda_{i} r_{i}}{s+\lambda_{i}}
$$

therefore any suitable method can be employed to estimate the poles and residues. This is offered as an alternative to using Procedure $\mathrm{X}$ and the NLSQ refinement

\subsubsection{Prediction of hysteresis loops}

Unbiased triangular voltages with a fixe amplitude of $63 \mathrm{~V}$ and three representative frequencies are applied to the PZT actuator. The input/output behavior is represented in Fig. 11, where it can be confirme that, for this system, linear phase lag dominates the loop shapes, since the simulated data corresponds to a model with essentially linear phase characteristics. The difference between simulated and predicted loops should provide any rate-independent hysteresis present in the system.

\subsection{Experimental results 3: model validation}

In this section, the numerical state-space model is confronted with a set of arbitrary test input signals. The simu-
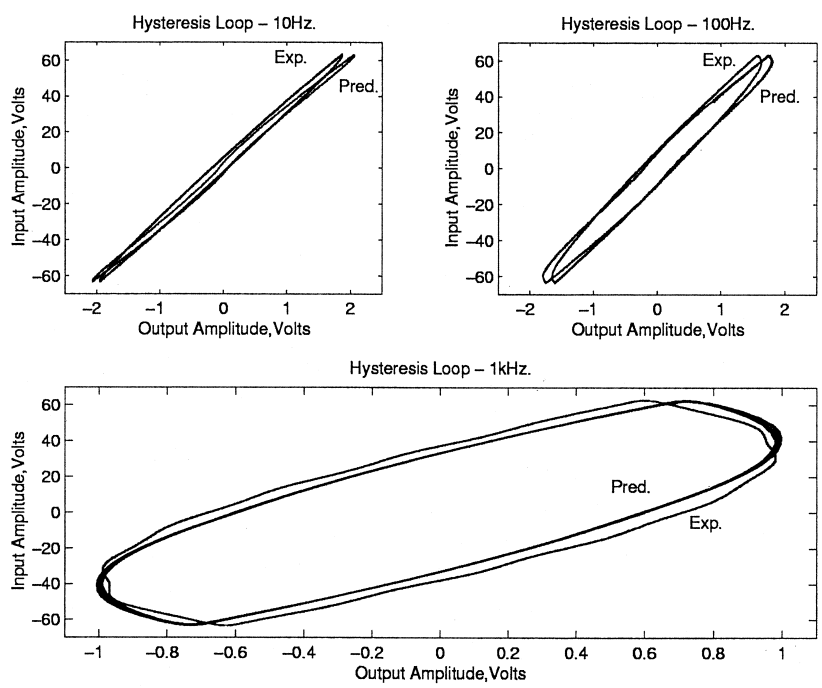

Fig. 11. Prediction of Hysteresis Loops.

lations are performed using the Matlab/Simulink package. The differential model is readily constructed from Eq. (6) using the identifie parameters. The state-space model can be compactly expressed as

$$
\left\{\begin{array}{c}
\dot{q}=A q+F(V) \\
\varepsilon=C q
\end{array}\right.
$$

where $A$ is a 7 -by-7 matrix given by

$$
A=\operatorname{diag}\left(-\lambda_{1},-\lambda_{2}, \ldots,-\lambda_{6}, 0\right)
$$

$C$ is a row vector of ones

$$
C=[1,1,1,1,1,1,1]
$$

and $F$ is the vector function given by

$$
\begin{aligned}
F(\sigma)= & {\left[\lambda_{1} r_{1}\left(\frac{V}{\mu}\right)^{p}, \lambda_{2} r_{2}\left(\frac{V}{\mu}\right)^{p}, \ldots,\right.} \\
& \left.\lambda_{6} r_{6}\left(\frac{V}{\mu}\right)^{p},\left(\frac{V}{\eta}\right)^{m}\right]
\end{aligned}
$$
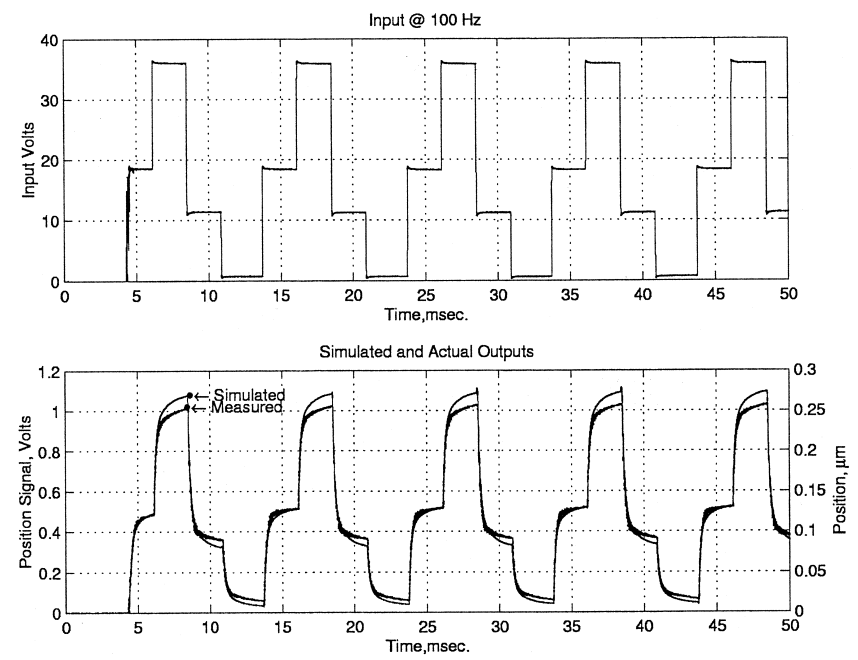

Fig. 12. Prediction of response to sequential voltage steps, $100 \mathrm{~Hz}$. 

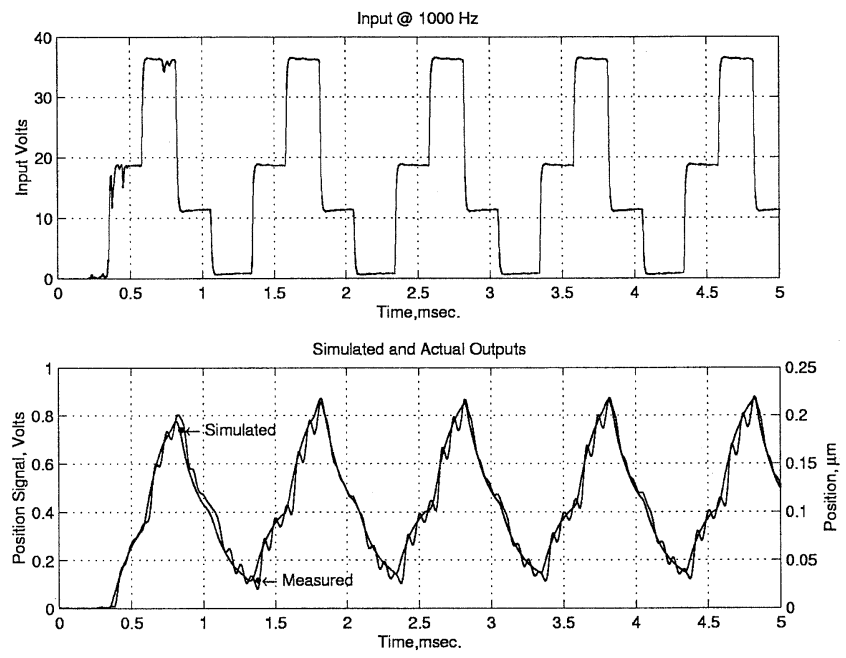

Fig. 13. Prediction of response to sequential voltage steps, $1 \mathrm{kHz}$.

Three kinds of signals are employed for validation: Sawtooth, staircase and a band-limited random input. Figs. 12 and 13 show the simulated and measured responses when the input is a staircase with nearly equal ascending steps and unequal descending steps, for low, medium and high frequencies. Figs. 14 and 15 show the simulated and measured responses to sawtooth excitation at $100 \mathrm{~Hz}$. and $1 \mathrm{kHz}$. respectively. It is seen that the model adequately follows the response, up to its design bandwidth. Additionally, the duration of some experiments was extended to the second range, to check the assumption of a time-invariant model, with positive results. The model is tested in its ability to predict the response to biased inputs. This is shown in Fig. 16 . The bias is adequately predicted by the model, despite the fact that the neglected resonance contributes to offset error. Finally, a zero-biased, uniform random input band limited at roughly $1 \mathrm{kHz}$ is applied. The results are depicted in Fig. 17. The general trend of the response is adequately tracked, however there exists a moderate error in the am-
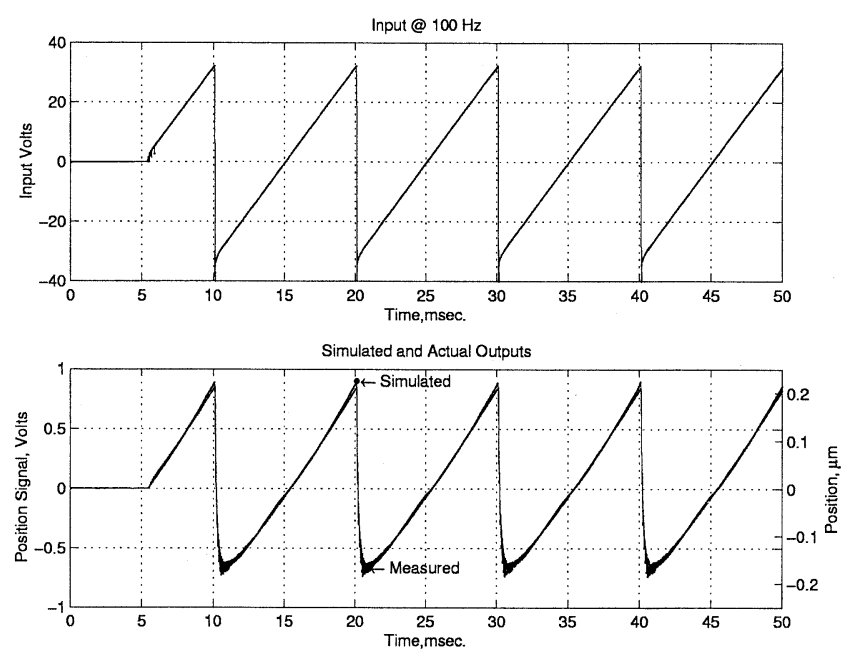

Fig. 14. Prediction of response to sawtooth voltage, $100 \mathrm{~Hz}$.
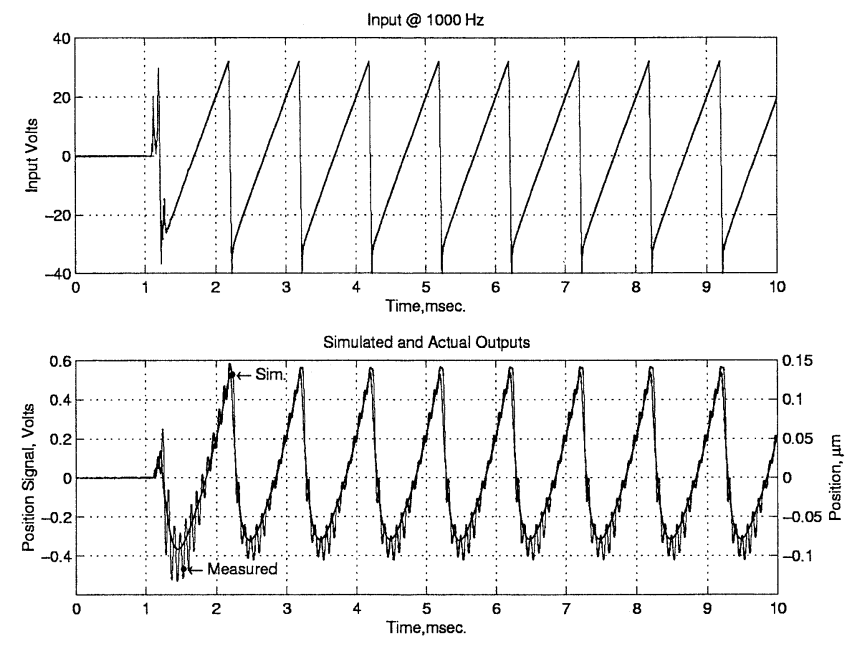

Fig. 15. Prediction of response to sawtooth voltage, $1 \mathrm{kHz}$.

plitudes. The prediction error relative to actual output, taken as a ratio of standard deviations is roughly 32 percent.

\section{Conclusions}

The model fit experimental data and predicts arbitrary responses with a good degree of accuracy. The results show that power laws are reasonable assumptions for the input functions, although the creep law introduces moderate errors. The model structure along with estimated parameters is shown to be consistent in the frequency and time domains. The model is also useful to reveal that dynamic hysteresis predominates in this particular PZT actuator, at least within the amplitude ranges under study. A shortcoming of the model is, perhaps, its high order. By observing the normalized frequency response in Fig. 10, it is obvious that a lower order model can be constructed, but at the expense of time-domain accuracy. For control applications, a lower
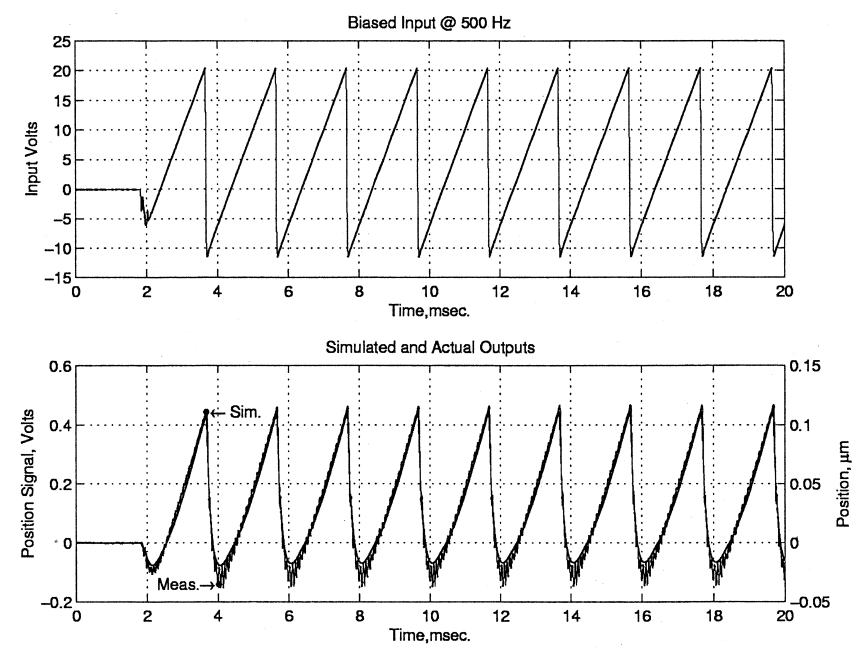

Fig. 16. Prediction of response to biased sawtooth voltage, $500 \mathrm{~Hz}$. 

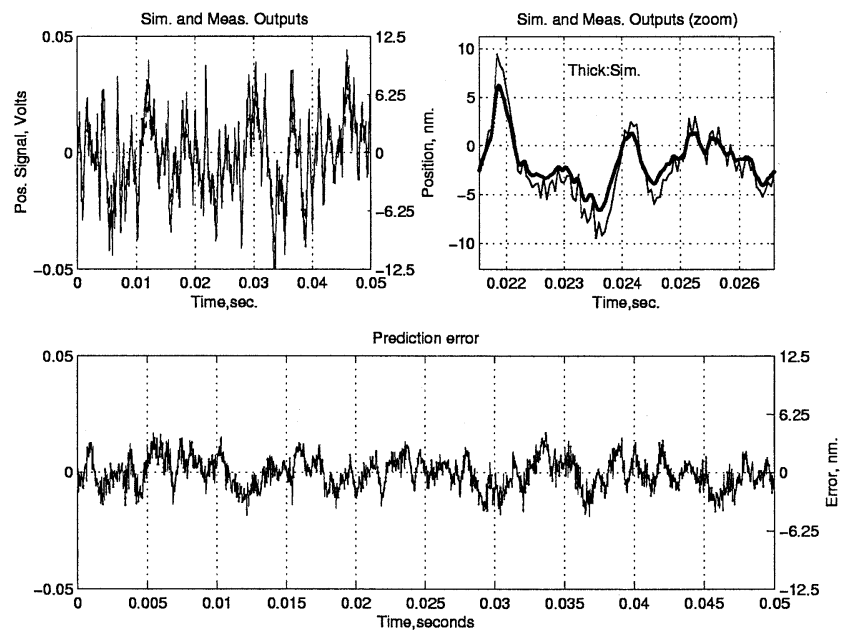

Fig. 17. Prediction of response to random input band limited at $1 \mathrm{kHz}$.

order model may be preferable, as long as it retains the essential characteristics in the frequency domain. If model reduction produces significan error, it is the robust control system's task to account for those errors and still produce desired closed-loop performance, which is the ultimate goal of this line of research. Among the qualitative shortcomings of the model is the fact that it predicts zero steady creep rate for zero stress input, regardless of past input history. This is a direct consequence of the strain-hardening theory employed for inducing dynamic behavior from the constant input response. Step-down experimental data shows, however, that the piezo actuator will creep even when the input is stepped down to zero from some constant voltage. This effect may be hard to capture in a differential model, for it involves not only past output history, but past input or input rate history. A more general nonlinear hereditary integral representation may be able to capture this effect, but an integral representation is inconvenient for control system design purposes, and it may be difficul to obtain an equivalent differential model. A more practically oriented drawback is that a full identificatio procedure for each actuator under study would be required, as it happens with all empirical models that are tailored to a specifi component. The model is a dynamic fi that allows the design of a control system. The main conclusion is that the proposed analogy does provide an adequate means to describe the nonlinearities present in this PZT tube actuator system. The PZT actuator has a quasi-linear behavior, at least in the range of amplitudes considered in this work. Closed-loop control, therefore, is straightforward to design and implement. The only difficultie expected in the design process are the same ones found in linear plants, namely, unmodeled resonance, sensor noise, and actuator saturations.

\section{Notes}

1. The authors would like to thank M.J. Klopfstein for his assistance with the use of Nanocut. The kind sup- port by the National Science Foundation, Division of Design, Manufacture and Industrial Innovation is gratefully acknowledged.

2. Manufactured by Staveley Sensors Inc., E. Hartford, Connecticut.

3. The PZT tube has a nonlinear static sensitivity.

4. In this context, transient represents a function of time that quickly converges to some steady value.

5. The NLSQ problem did not include this constraint.

\section{References}

[1] Smith ST, Chetwynd DG. Foundations of ultraprecision mechanism design. Amsterdam: Gordon and Breach Science Publishers, 1997.

[2] Lucca DA, Hocken RJ, et al. An ultra-precision instrument for controlled nanoindenting and nanocutting. Progress in Precision Engineering and Nanotechnology, Proceedings of the 9th International Precision Engineering Seminar/4th International Conference on Ultraprecision in Manufacturing Engineering Braunschweig. Germany, 1997. p. 725-28.

[3] Tamer N, Dahleh M. Feedback control of piezoelectric tube scanners. Proceedings of the 33rd Conference of Decision and Control. 1994. p. $1826-31$.

[4] Tsuchiura KM, et al. Disk system with sub-actuators for fin head displacement. U.S. Patent 5189578, Feb. 23, 1993.

[5] IEEE Standard on Piezoelectricity, ANSI/IEEE Std, 176-1987.

[6] Kaizuka H, Siu B. A simple way to reduce hysteresis and creep when using piezoelectric actuators. Jap J Appl Phys 1988;27(5):773-6.

[7] Low TS, Guo W. Modeling of a three-layer piezoelectric bimorph beam with hysteresis. J Microelectromechanical Syst. 1995;4(4):230-7.

[8] Morgan Matroc Inc. Electro Ceramics Division, Bedford, OH, Guide to Modern Piezoelectric Ceramics, March 1993.

[9] Main JA, Garcia E. Piezoelectric Stack Actuators and Control System Design: Strategies and Pitfalls. J Guid Cont and Dynam 1997;20(3): $479-85$.

[10] Campos Rubio JC, et al. Comparison of control strategies for piezoelectric and magnetostrictive micropositioning devices for ultra-precision machining. ASPE Proc 1996;14:294-9.

[11] Tiersten HF. On the nonlinear equations of thermoelectroelasticity. Int J Eng Sci 1971;9:587.

[12] Goldfarb M, Celanovic N. Modeling piezoelectric stack actuators for control of micromanipulation. IEEE Con Syst Mag 1997;17(3):6979 .

[13] Zhang QM, Wang H, Cross LE. Piezoelectric tubes and tubular composites for actuator and sensor applications. J Mat Sci 1993;28: 1962-8.

[14] Vieira S. The behavior and calibration of some piezoelectric ceramics used in the STM. IBM J Res Dev 1986;30A(5):553-6.

[15] Tschoegl NW. The phenomenological theory of linear viscoelastic behavior. Berlin: Springer-Verlag, 1989.

[16] Marin J, Pao YH. An analytical theory of the creep deformation of materials. J Appl Mech 1953;20.

[17] Penny RK, Marriot DL. Design for creep. London: Chapman \& Hall, 1995.

[18] Shames IH, Cozzarelli FA. Elastic and inelastic stress analysis. Washington DC: Taylor \& Francis, 1997.

[19] Jouaneh M, Tian H. Accuracy enhancement of a piezoelectric actuator with hysteresis. JAPAN/USA Symposium on Flexible Automation, vol. 1, pp. 631-7, ASME 1992.

[20] Marin J. Mechanical behavior of engineering materials. Prentice-Hall, 1962.

[21] Ge, Ping, Jouaneh. Modeliing hysteresis in piezoceramic actuators. Prec Eng 1995;17:211-21. 